\title{
Consensus Problems in Networks of Agents with Switching Topology and Time-Delays
}

\author{
Reza Olfati Saber Richard M. Murray \\ Control and Dynamical Systems \\ California Institute of Technology \\ e-mails: $\{$ olfati,murray\}@cds.caltech.edu
}

April 23, 2003

Keywords: consensus problems, networks of autonomous agents, switching systems, graph Laplacians, balanced graphs, communication time-delays, algebraic graph theory.

\begin{abstract}
In this paper, we discuss consensus problems for a network of dynamic agents with fixed and switching topologies. We analyze three cases: i) networks with switching topology and no time-delays, ii) networks with fixed topology and communication time-delays, and iii) max-consensus problems (or leader determination) for groups of discrete-time agents. In each case, we introduce a linear/nonlinear consensus protocol and provide convergence analysis for the proposed distributed algorithm. Moreover, we establish a connection between the Fiedler eigenvalue of the information flow in a network (i.e. algebraic connectivity of the network) and the negotiation speed (or performance) of the corresponding agreement protocol. It turns out that balanced digraphs play an important role in addressing average-consensus problems. We introduce disagreement functions that play the role of Lyapunov functions in convergence analysis of consensus protocols. A distinctive feature of this work is to address consensus problems for networks with directed information flow. We provide analytical tools that rely on algebraic graph theory, matrix theory, and control theory. Simulations are provided that demonstrate the effectiveness of our theoretical results.
\end{abstract}

\section{Introduction}

Distributed decision-making for coordination of networks of dynamic agents has attracted several researchers in recent years. This is partly due to broad applications of multi-agent systems in many areas including cooperative control of unmanned air vehicles (UAVs), flocking 
of birds [22, 24, 23], schooling for underwater vehicles, distributed sensory networks, attitude alignment of clusters of satellites, and congestion control in communication networks [21].

Agreement problems have a long history in the field of computer science, particularly in automata theory and distributed computation [15]. In many applications involving multiagent/multi-vehicle systems, groups of agents need to agree upon certain quantities of interest. Such quantities might or might not be related to the motion of the individual agents. As a result, it is important to address agreement problems in their general form for networks of dynamic agents with directed information flow under link failure and creation (i.e. variable network topology).

Our main contribution in this paper is to define and address consensus problems under a variety of assumptions on the network topology (being fixed or variable), presence or lack of communication time-delays, and boundedness of the inputs of dynamic agents. In each case, we provide convergence analysis and for linear protocols establish direct connections between performance and robustness of a consensus protocol and the properties of graph Laplacian of the information flow in the network.

In the past, a number of researchers have worked in problems that are essentially different forms of agreement problems with differences regarding the types of agent dynamics, the properties of the graphs, and the names of the tasks of interest. In [25, 7, 6], graph Laplacians are used for the task of formation stabilization for groups of agents with linear dynamics. Their method for formation stabilization has not yet been extended to systems with nonlinear dynamics that are not feedback linearizable. Special cases of this approach are known as leader-follower type architectures and have been widely used by numerous researchers $[19,4$, 14]. In [18], graph Laplacians are used in the context of dynamic graph theory. In [24, 23], flocking and heading angle alignment for multiple particles is analyzed from the point of view of statistical mechanics and a phase transition phenomenon is observed that occurs the information flow in the network becomes connected. The work in [13] focuses on attitude alignment for undirected graphs in which the agents have simple dynamics motivated by the model used in [24]. It is claimed that the connectivity of the graph on average is sufficient for convergence of the heading angles of the agents. In [20], the authors addressed convergence of linear and nonlinear protocols for networks with undirected graphs in presence or lack of communication time-delays. Theoretically, analyzing consensus on directed graphs is more challenging and is considered in the present paper.

In this paper, we provide convergence analysis of consensus protocol for a network of integrators with a directed information flow and fixed or switching topology. Our analysis relies on several tools from algebraic graph theory $[1,11]$ and matrix theory [12]. We establish a connection between the performance of the linear consensus protocol and the Fiedler eigenvalue of graph Laplacian of a graph called the mirror graph (that is closely-related to the original directed graph).

It turns out that a class of directed graphs called balanced graphs have a crucial role in derivation of an invariant quantity and a Lyapunov function for convergence analysis of average-consensus problems on directed graphs. This Lyapunov function is a measure of group disagreement in the network. We show that a directed graph solves the average- 
consensus problem using a certain protocol if and only if it is balanced. Furthermore, we use properties of balanced networks to analyze the convergence of an agreement protocol for networks with switching topology. This variation of the network topology is usually due to link failures or creations in networks with mobile nodes. We introduce a common Lyapunov function that guarantees asymptotic convergence to a group decision value in a network with switching information flow.

Building on these basic results, we provide a number of extensions. We discuss the effects of communication time-delays in solving consensus problems. For undirected graphs, we address average-consensus problem under the constraint that the inputs of all agents are bounded. This leads to analyzing a nonlinear protocol. Using a example of a network with three nodes, we demonstrate that analyzing the convergence of this protocol for general digraphs is a challenging open problem. Finally, we pose and address the max-consensus problem that is useful for determining a superior or leader in a group of agents in a distributed way.

Simulation results are provided that demonstrate our theoretical predictions and show the novel analytical tools that we propose are effective.

An outline of this paper is as follows. In Section 2, we define consensus problems. In Section 3, we give two protocols. Some background on algebraic graph theory and properties of graph Laplacians are provided in Section 4. A counterexample is given in Section 5 that shows not every digraph solves an average-consensus problem. In Section 6, our main results on networks with switching topology are presented. Average-consensus problems for networks with communication time-delays is discussed in Section 7. In Section 8, our result on max-consensus problem is given. The simulation results are presented in Section 9. Finally, in Section 10, concluding remarks are stated.

\section{Consensus Problems}

Let $G=(\mathcal{V}, \mathcal{E}, \mathcal{A})$ be a weighted directed graph (or digraph) with $n$ nodes and a weighted adjacency matrix $\mathcal{A}=\left[a_{i j}\right]$ where $a_{i j} \geq 0$ for all $i, j \in \mathcal{I}=\{1,2, \ldots, n\}$ with $i \neq j$. Here, $\mathcal{V}$ denotes the set of vertices $v_{i}$ and $\mathcal{E}$ denotes the set of edges $i j$ (or $\left(v_{i}, v_{j}\right)$ ) of the graph $G$. The set of neighbors of node $i$ is denoted by $N_{i}=\left\{i j \in \mathcal{E}: a_{i j}>0\right\}$. We call any subset of nodes $J$ a cluster. The set of neighbors of a cluster $J \subset \mathcal{I}$ is defined by

$$
N_{J}:=\cup_{i \in J} N_{i}=\{j \in \mathcal{I}: i \in J, i j \in \mathcal{E}\}
$$

Let $x_{i} \in \mathbb{R}$ denote the value of node $i$. We refer to $G_{x}=(\mathcal{V}, \mathcal{E}, \mathcal{A}, x)$ with $x=$ $\left(x_{1}, \ldots, x_{n}\right)^{T}$ as an algebraic graph or (static) network with value $x \in \mathbb{R}^{n}$ and information flow $G=(\mathcal{V}, \mathcal{E}, \mathcal{A})$. The value of a node might represent physical quantities including attitude, position, temperature, voltage, and so on. We say nodes $i$ and $j$ in a network agree if and only $x_{i}=x_{j}$. We say all the nodes in a network have reached a consensus if and only

if $x_{i}=x_{j}$ for all $i, j \in \mathcal{I}, i \neq j$. Whenever the nodes of a network are in agreement, the common value of all nodes is called the (group) decision value. 
Suppose each node of a graph is a dynamic agent with dynamics

$$
\dot{x}_{i}=f\left(x_{i}, u_{i}\right), \quad i \in \mathcal{I} .
$$

A dynamic graph (or dynamic network) is defined as a 4-tuple $G_{x(t)}=(\mathcal{V}, \mathcal{E}, \mathcal{A}, x(t))$ together with dynamics $\dot{x}=F(x, u)$ where $x$ denotes the state of the dynamic graph and $F(x, u)$ is the column-wise concatenation of the elements $F_{i}(x, u)=f\left(x_{i}, u_{i}\right)$.

Let $\chi: \mathbb{R}^{n} \rightarrow \mathbb{R}$ be a function of $n$ variables $x_{1}, \ldots, x_{n}$. The $\chi$-consensus problem in a dynamic graph is a distributed way to calculate $\chi(x(0))$ by applying inputs $u_{i}$ that only depend on the values of node $i$ and its neighbors. We say a protocol

$$
u_{i}=k_{i}\left(x_{j_{1}}, \ldots, x_{j_{m_{i}}}\right)
$$

with $j_{1}, \ldots, j_{m_{i}} \in\{i\} \cup N_{i}$ and $m_{i} \leq n$ asymptotically solves the $\chi$-consensus problem if and only if there exists an asymptotically stable equilibrium $x^{*}$ of $\dot{x}=F(x, k(x))$ such that $x_{i}^{*}=\chi(x(0))$ for all $i \in \mathcal{I}$. We are interested in solving the $\chi$-consensus problem in a distributed fashion in which no node is connected to all other nodes (i.e. $m_{i}<n$ for all $i$ ).

The special cases with $\chi(x)=\operatorname{Ave}(x)=\frac{1}{n}\left(\sum_{i=1}^{n} x_{i}\right), \chi(x)=\operatorname{Max}(x)=\max _{i} x_{i}$, and $\chi(x)=\operatorname{Min}(x)=\min _{i} x_{i}$ are called average-consensus, max-consensus, and min-consensus, respectively due to their broad applications in distributed decision-making for multi-agent systems.

Solving average-consensus problem is an an example of distributed computation of a linear function $\chi(x)=\operatorname{Ave}(x)$ using a network of dynamic systems. This is more challenging than just reaching a general agreement.

\section{Consensus Protocols}

In this section, we present three consensus protocols that solve agreement problems in a network of continuous-time (CT) integrator agents with dynamics

$$
\dot{x}_{i}(t)=u_{i}(t)
$$

or agents with discrete-time (DT) model

$$
x_{i}(k+1)=x_{i}(k)+\epsilon u_{i}(k)
$$

and step-size $\epsilon>0$. In this paper, we consider three scenarios:

i) Fixed or switching topology and zero communication time-delay: We use the following linear consensus protocol:

$$
u_{i}=\sum_{j \in N_{i}} a_{i j}\left(x_{j}-x_{i}\right)
$$

where the set of neighbors $N_{i}=N_{i}(G)$ of node $i$ is variable in networks with switching topology. 
ii) Fixed topology $G=(\mathcal{V}, \mathcal{E}, \mathcal{A})$ and communication time-delay $\tau_{i j}>0$ corresponding to the edge $i j \in \mathcal{E}$ : We use the following linear time-delayed consensus protocol:

$$
u_{i}(t)=\sum_{j \in N_{i}} a_{i j}\left[x_{j}\left(t-\tau_{i j}\right)-x_{i}\left(t-\tau_{i j}\right)\right]
$$

The derivation of each of the aforementioned two protocols (A1) and (A2) becomes apparent during the convergence analysis that will be presented for each protocol later on. We show that in each case, consensus can is asymptotically reached. Moreover, we provide sufficient and necessary conditions on the directed information flow in the network so that average-consensus, max-consensus, and min-consensus can be achieved. Furthermore, we provide results on performance and algorithmic robustness of these consensus protocols.

Remark 1. For an undirected network with fixed topology, both protocols solve the averageconsensus problem with appropriate technical conditions on delays. The challenge is to address similar consensus problems for networks with directed graphs and switching topology. In multi-agent flocking [22], the information flow is usually directed and the topology of the network goes through changes that are discrete-event type in the nature.

Given Protocol (A1), the state of a network of continuous-time integrator agents evolves according to the following linear system

$$
\dot{x}(t)=-L x(t)
$$

where $L$ is called the graph Laplacian induced by the information flow $G$ and is defined by

$$
l_{i j}= \begin{cases}\sum_{k=1, k \neq i}^{n} a_{i k}, & j=i \\ -a_{i j}, & j \neq i\end{cases}
$$

The properties of graph Laplacian is one of the main areas of research in algebraic graph theory and is discussed in Section 4.

In a network with switching topology, convergence analysis of Protocol (A1) is equivalent to stability analysis for a hybrid system

$$
\dot{x}(t)=-L_{k} x(t), \quad k=s(t)
$$

where $L_{k}=\mathcal{L}\left(G_{k}\right)$ is the Laplacian of $G_{k}, s(t): \mathbb{R} \rightarrow \mathcal{I}_{\Gamma} \subset \mathbb{Z}$ is a switching signal, and $\Gamma \ni G_{k}$ is a finite collection of digraphs (of order $n$ ) with the index set $\mathcal{I}_{\Gamma}$. Later, we will see that $\Gamma$ is a relatively large set for $n \gg 1$. The task of stability analysis for the hybrid system in (8) is rather challenging partly because, in general, the product of two Laplacian matrices do not commute.

For agents with discrete-time models, applying protocol (A1) gives the following discretetime network dynamics

$$
x(k+1)=P_{\epsilon} x(k)
$$


with

$$
P_{\epsilon}=I-\epsilon L .
$$

Let $d_{\text {max }}=\max _{i} l_{i i}$, then for $\epsilon \in\left(0,1 / d_{\max }\right), P_{\epsilon}$ is a nonnegative and stochastic matrix that we call Perron matrix, i.e. $P_{\epsilon}$ in element-wise nonnegative and all of its row sums are 1.

The convergence analysis of Protocol (A1) for discrete-time agents heavily relies on the theory of nonnegative matrices $[10,12,16]$ and will be discussed in a separate paper. Our approach presents a Lyapunov-based convergence analysis for agreement in networks with discrete-time models. This is different than the approach pursued in the work by Jadbabaie et al. which strongly relies on matrix theoretic properties and infinite right-convergent products (RCP) of stochastic matrices [2, 3].

\section{Algebraic Graph Theory: Properties of Laplacians}

In this section, we introduce some basic concepts and notation in graph theory that will be used throughout the paper. More information is available in [11, 5]. A comprehensive survey on properties of Laplacians of undirected graphs can be found in [17]. However, we need to work with Laplacians of directed graphs that the basic properties cannot be found in graph theory literature and will be stated here.

Let $G=(\mathcal{V}, \mathcal{E}, \mathcal{A})$ be a weighted directed graph (or digraph) with $n$ nodes. The in-degree and out-degree of node $v_{i}$ are, respectively, defined as follows:

$$
\operatorname{deg}_{\text {in }}\left(v_{i}\right)=\sum_{j=1}^{n} a_{j i}, \operatorname{deg}_{\text {out }}\left(v_{i}\right)=\sum_{j=1}^{n} a_{i j} .
$$

For an ordinary graph with adjacency matrix $\mathcal{A}$ that has binary elements in the set $\{0,1\}$, $\operatorname{deg}_{\text {out }}\left(v_{i}\right)=\left|N_{i}\right|$. The degree matrix of $G$ is a diagonal matrix denoted by $\Delta=\left[\Delta_{i j}\right]$ where $\Delta_{i j}=0$ for all $i \neq j$ and $\Delta_{i i}=\operatorname{deg}_{\text {out }}\left(v_{i}\right)$. The (weighted) graph Laplacian matrix associated with $G$ is defined as

$$
L=\mathcal{L}(G)=\Delta-\mathcal{A} .
$$

This is consistent with the definition of the elements of $L$ in (7).

Remark 2. The graph Laplacian $L$ does not depend of the diagonal elements $a_{i i}$ of the adjacency matrix of $G$ corresponding to the weights of loops $\left(v_{i}, v_{i}\right)$ (i.e. cycles of length one). Depending on the context, without loss of generality we might assume $a_{i i}=0$ for all $i$. In a restricted part of this work that makes use of graphs with loops that their weight cannot be discarded.

We sometimes use $\mathcal{L}(\mathcal{A})=\mathcal{L}(G)$ to denote the Laplacian of graph $G$. By definition, every row sum of the Laplacian matrix is zero. Therefore, graph Laplacian always has a zero eigenvalue (i.e. $\operatorname{rank}(L) \leq n-1$ ) corresponding to a right eigenvector

$$
w_{r}=\mathbf{1}=(1,1, \ldots, 1)^{T}
$$


with identical nonzero elements.

A digraph is called strongly connected if and only if any two distinct nodes of the graph can be connected via a path that respects the orientation of the edges of the digraph. The following theorem establishes a direct relation between the SC property of a digraph and the rank of its Laplacian.

Theorem 1. Let $G=(\mathcal{V}, \mathcal{E}, \mathcal{A})$ be a weighted digraph with Laplacian L. Then, $G$ is strongly connected if and only if $\operatorname{rank}(L)=n-1$.

Proof. See Section A.1 in the Appendix.

Remark 3. For an undirected graph $G$, the proof of Theorem 1 is easy and can be found in $[1,11]$.

Remark 4. For an undirected graph $G$ with a symmetric adjacency matrix $\mathcal{A}$, the graph Laplacian $L$ is symmetric and positive semidefinite. The Laplacian potential associated with $G$ is defined in [20] as follows

$$
\Phi_{G}(x)=x^{T} L x=\frac{1}{2} \sum_{i j \in \mathcal{E}}\left(x_{j}-x_{i}\right)^{2}
$$

Here is a short proof of Theorem for an undirected graph: Assume $L x=0$ for $x \in \mathbb{R}^{n}$. Then $x^{T} L x=0$ and $x_{j}=x_{i}$ for all the edges $i j \in \mathcal{E}$. If the graph is connected, this means that all nodes agree and $x_{1}=\ldots=x_{n}$. Thus, $\operatorname{rank}(L)=n-1$. Since $\Phi_{G}(x)=0$ for a connected graph implies all nodes are in agreement, $\Phi_{G}(x)$ provides a meaningful quantification of disagreement in a group of agents.

For an undirected graph $G$ that is connected the following well-known property holds [11]:

$$
\min _{\substack{x \neq 0 \\ \mathbf{1}^{T} x=0}} \frac{x^{T} L x}{\|x\|^{2}}=\lambda_{2}(L)
$$

The proof follows from a special case of Courant-Fischer Theorem in [12]. We will later establish a connection between $\lambda_{2}(\hat{L})$ with $\hat{L}=\left(L+L^{T}\right) / 2$, called the Fiedler eigenvalue of $\hat{L}[8,9]$, and the performance (i.e. worst-case negotiation speed) of a linear agreement protocol.

The key in the stability analysis of system (6) is in the spectral properties of graph Laplacian. The following result is well-known (e.g. see [17]) and is based on Geršgorin disk theorem [12].

Theorem 2. (spectral localization) Let $G=(\mathcal{V}, \mathcal{E}, \mathcal{A})$ be a digraph with the Laplacian $L$. Denote the maximum node out-degree of $G$ by $d_{\text {max }}(G)=\max _{i} \operatorname{deg}_{\text {out }}\left(v_{i}\right)$. Then, all the eigenvalues of $L=\mathcal{L}(G)$ are located in the following disk

$$
D(G)=\left\{z \in \mathbb{C}:\left|z-d_{\max }(G)\right| \leq d_{\max }(G)\right\}
$$

centered at $z=d_{\max }(G)+0 j$ in the complex plane (see Figure 1). 


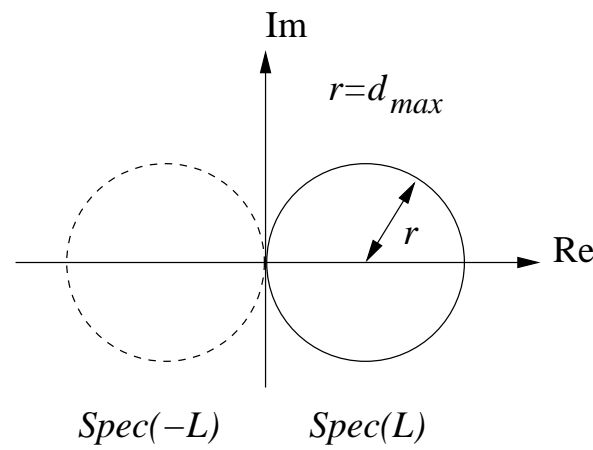

Figure 1: A demonstration of Geršgorin Theorem applied to graph Laplacian.

Proof. Based on the Geršgorin disk theorem, all the eigenvalues of $L=\left[l_{i j}\right]$ are located in the union of the following $n$ disks

$$
D_{i}=\left\{z \in \mathbb{C}:\left|z-l_{i i}\right| \leq \sum_{j \in \mathcal{I}, j \neq i}\left|l_{i j}\right|\right\} .
$$

But $l_{i i}=\Delta_{i i}$ and

$$
\sum_{j \in \mathcal{I}, j \neq i}\left|l_{i j}\right|=\operatorname{deg}_{\text {out }}\left(v_{i}\right)=\Delta_{i i} .
$$

Thus, $D_{i}=\left\{z \in \mathbb{C}:\left|z-\Delta_{i i}\right| \leq \Delta_{i i}\right\}$. On the other hand, all these $n$ disks are contained in the largest disk $D(G)$ with radius $d_{\max }(G)$. Clearly, all the eigenvalues of $-L$ are located in the disk $D^{\prime}(G)=\left\{z \in \mathbb{C}:\left|z+d_{\max }(G)\right| \leq d_{\max }(G)\right\}$ that is the mirror image of $D(G)$ with respect to the imaginary axis.

Here is an immediate corollary and the first convergence proof for protocol (A1) for a directed network.

Corollary 1. Consider a network of integrators $\dot{x}_{i}=u_{i}$ where each node applies protocol (A1). Assume $G$ is a strongly connected digraph. Then, protocol (A1) globally asymptotically solves a consensus problem.

Proof. Since $G$ is strongly connected, $\operatorname{rank}(L)=n-1$ and $L$ has a simple eigenvalue at zero. Based on Theorem 2, the rest of the eigenvalues of $-L$ have negative real-parts and therefore the linear system in (6) is stable. On the other hand, any equilibrium $x^{*}$ of (6) is a right eigenvector of $L$ associated with $\lambda=0$. Since the eigenspace associated with the zero eigenvalue is one-dimensional, there exists an $\alpha \in \mathbb{R}$ such that $x^{*}=\alpha \mathbf{1}$, i.e. $x_{i}^{*}=\alpha$ for all $i$.

Keep in mind that Corollary 1 does not guarantee whether the decision value $\alpha$ of each node is equal to Ave $(x(0))$ or not. In other words, Corollary 1 does not necessarily address the average-consensus problem. 


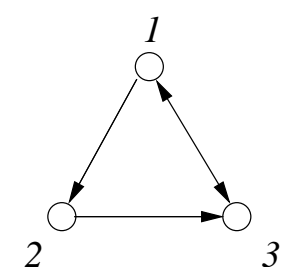

Figure 2: A connected digraph of order 3 that does not solve the average-consensus problem using Protocol (A1).

\section{A Counterexample for Average-Consensus}

A sufficient condition for the decision value $\alpha$ of each node in the proof of Corollary 1 to be equal to $\operatorname{Ave}(x(0))$ is that $\sum_{i=1}^{n} u_{i} \equiv 0$. If $G$ is undirected (i.e. $a_{i j}=a_{j i}>0, \forall i, j: a_{i j} \neq 0$ ), automatically the condition $\sum_{i=1}^{n} u_{i}=0, \forall x$ holds and Ave $(x(t))$ is an invariant quantity [20]. However, this property does not hold for a general digraph.

A simple counterexample is a strongly connected digraph of order $n=3$, shown in Figure 2, with weights in $\{0,1\}$ and the following sets of vertices and edges:

$$
\mathcal{V}=\{1,2,3\}, \mathcal{E}=\{12,23,31,13\}
$$

For the digraph $G=(\mathcal{V}, \mathcal{E}), \sum_{i=1}^{3} u_{i}=x_{3}-x_{1}$. Thus, if nodes 1 and 3 disagree, the property $\sum_{i=1}^{3} u_{i}=0$ does not hold for all $x$. On the other hand, the reader can verify that for this example

$$
L=\left[\begin{array}{ccc}
2 & -1 & -1 \\
0 & 1 & -1 \\
-1 & 0 & 1
\end{array}\right]
$$

and $x_{i}^{*}=\left[x_{1}(0)+x_{2}(0)+2 x_{3}(0)\right] / 4$ (this is due to Theorem 3 ). This decision value is apparently in the convex hull of all the $x_{i}(0)$ 's but it is different from Ave $(x(0))$ if and only if $x_{1}(0)+x_{2}(0) \neq 2 x_{3}(0)$. As a result, for all initial conditions satisfying $x_{1}(0)+x_{2}(0) \neq 2 x_{3}(0)$, Protocol (A1) does not solve the average-consensus problem but still all nodes reach an agreement. This motivates us to characterize the class of all digraphs that solve the averageconsensus problem.

Before presenting our first main result, we need to provide a limit theorem for exponential matrices of the form $\exp (-L t)$. This is because the solution of $(6)$ is given by

$$
x(t)=\exp (-L t) x(0)
$$

and by explicit calculation of $\exp (-L t)$, we can obtain the decision value of each node after reaching consensus for a general digraph. The following theorem is closely related to a famous limit theorem in the theory of nonnegative matrices known as Perron-Frobenius Theorem [12]. 
Notation. Following the notation in [12], we denote the set of $m \times n$ real matrices by $M_{m, n}$ and the set of square $n \times n$ matrices by $M_{n}$. Furthermore, throughout this paper, the right and left eigenvectors of the Laplacian $L$ associated with $\lambda_{1}=0$ are denoted by $w_{r}$ and $w_{l}$, respectively.

Theorem 3. Assume $G$ is a strongly connected digraph with Laplacian $L$ satisfying $L w_{r}=0$, $w_{l}^{T} L=0$, and $w_{l}^{T} w_{r}=1$. Then

$$
R=\lim _{t \rightarrow+\infty} \exp (-L t)=w_{r} w_{l}^{T} \in M_{n}
$$

Proof. Let $A=-L$ and let $J$ be the Jordan form associated with $A$, i.e. $A=S J S^{-1}$. We have $\exp (A t)=S \exp (J t) S^{-1}$ and as $t \rightarrow+\infty, \exp (J t)$ converges to a matrix $Q=\left[q_{i j}\right]$ with a single nonzero element $q_{11}=1$. The fact that other blocks in the diagonal of $\exp (J t)$ vanish is due to the property that $\operatorname{Re}\left(\lambda_{k}(A)\right)<0$ for all $k \geq 2$ where $\lambda_{k}(A)$ is the $k$ th largest eigenvalue of $A$ in terms of magnitude $\left|\lambda_{k}\right|$. Notice that $R=S Q S^{-1}$. Since $A S=S J$ the first column of $S$ is $w_{r}$. Similarly, $S^{-1} A=J S^{-1}$ that means the first row of $S^{-1}$ is $w_{l}^{T}$. Due to the fact that $S^{-1} S=I, w_{l}$ satisfies the property $w_{l}^{T} w_{r}=1$ as stated in the question. A straightforward calculation shows that $R=w_{r} w_{l}^{T} \in M_{n}$.

\section{Networks with Fixed or Switching Topology}

In this section, we present three of our main results: i) characterization of all connected digraphs that solve average-consensus problem using Protocol (A1), and ii) the relation between the performance of Protocol (A1) and the Fiedler eigenvalue (i.e. algebraic connectivity) of graphs, and iii) robust agreement under switching topology due to link failure/creation.

\subsection{Balanced Graphs and Average-Consensus on Digraphs}

The following class of digraphs turns out to be instrumental in solving average-consensus problems:

Definition 1. (balanced graphs) We say the node $v_{i}$ of a digraph $G=(\mathcal{V}, \mathcal{E}, \mathcal{A})$ is balanced if and only if its in-degree and out-degree are equal, i.e. $\operatorname{deg}_{\text {out }}\left(v_{i}\right)=\operatorname{deg}_{\text {in }}\left(v_{i}\right)$. A graph $G=(\mathcal{V}, \mathcal{E}, \mathcal{A})$ is called balanced if and only if all of its nodes are balanced, i.e. $\sum_{j} a_{i j}=$ $\sum_{j} a_{j i}, \forall i$.

Example 1. Any undirected graph is balanced. Furthermore, the digraphs shown in Figure 3 are all balanced.

Here is our first main result: 


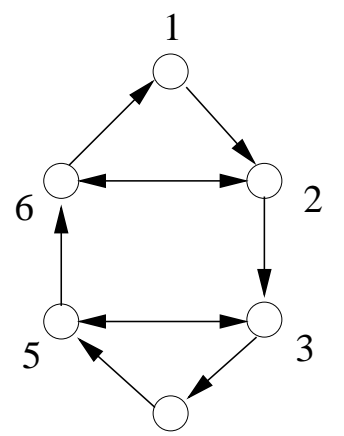

(a)

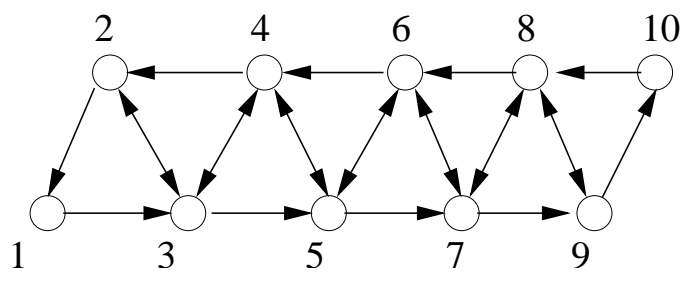

(b)

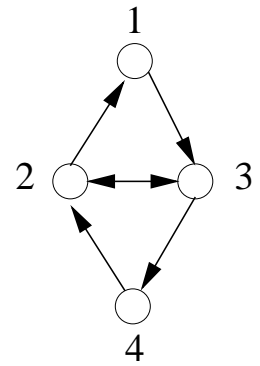

(c)

Figure 3: Three examples of balanced graphs.

Theorem 4. Consider a network of integrators with directed information flow $G=(\mathcal{V}, \mathcal{E}, \mathcal{A})$ that is strongly connected. Then, protocol (A1) globally asymptotically solves the averageconsensus problem using if and only if $G$ is balanced.

Proof. The proof follows from Theorems 5 and 6 , below.

Remark 5. According to Theorem 4, if a graph is not balanced, then protocol (A1) does not necessarily solve the average consensus-problem. This assertion is consistent with the counterexample given in Figure 2.

Theorem 5. Consider a network of integrator agents with a digraph $G=(\mathcal{V}, \mathcal{E}, \mathcal{A})$ that is strongly connected. Then, protocol (A1) globally asymptotically solves the average-consensus problem if and only if $\mathbf{1}^{T} L=0$.

Proof. From Theorem 3, with $w_{r}=\frac{1}{\sqrt{n}} \mathbf{1}$ we obtain

$$
x^{*}=\lim _{t \rightarrow+\infty} x(t)=R x_{0}=w_{r}\left(w_{l}^{T} x_{0}\right)=\frac{1}{\sqrt{n}}\left(w_{l}^{T} x_{0}\right) \mathbf{1} .
$$

This implies Protocol 1 globally exponentially solves a consensus problem with the decision value $\frac{1}{\sqrt{n}}\left(w_{l}^{T} x_{0}\right)$ for each node. If this decision value is equal to $\operatorname{Ave}\left(x_{0}\right), \forall x_{0} \in \mathbb{R}^{n}$, then necessarily $\frac{1}{\sqrt{n}} w_{l}=\frac{1}{\sqrt{n}}$, i.e. $w_{l}=w_{r}=\frac{1}{\sqrt{n}} \mathbf{1}$. This implies that $\mathbf{1}$ is the left eigenvector of $L$. To prove the converse, assume that $\mathbf{1}^{T} L=0$. Let us take $w_{r}=\frac{1}{\sqrt{n}} \mathbf{1}, w_{l}=\beta \mathbf{1}$ with $\beta \in \mathbb{R}, \beta \neq 0$. From condition $w_{l}^{T} w_{r}=1$, we get $\beta=\frac{1}{\sqrt{n}}$ and $w_{l}=\frac{1}{\sqrt{n}} \mathbf{1}$. This means that the decision value for every node is $\frac{1}{\sqrt{n}}\left(w_{l}^{T} x_{0}\right)=\frac{1}{n} \mathbf{1}^{T} x_{0}=\operatorname{Ave}\left(x_{0}\right)$.

Corollary 2. Assume all the conditions in Theorem 5 hold. Suppose L has a left eigenvector $\gamma=\left(\gamma_{1}, \ldots, \gamma_{n}\right)^{T}$ associated with $\lambda=0$ that is a nonnegative vector in $\mathbb{R}^{n}$ (i.e. a vector with non-negative elements) satisfying $\sum_{i} \gamma_{i}>0$. Then, the group decision value after reaching consensus is given by

$$
\alpha=\frac{\sum_{i} \gamma_{i} x_{i}(0)}{\sum_{i} \gamma_{i}}
$$


i.e. the decision value is in the convex hull of initial values of the nodes.

Proof. We have $\gamma^{T} L=0$ and thus $\gamma^{T} u \equiv 0$ (because $u=-L x$ ). Therefore, $\beta=\gamma^{T} x$ is an invariant quantity. Assume, the digraph $G$ is not balanced. Then, still an agreement is asymptotically reached. Let $\alpha$ be the decision value of all nodes after reaching consensus. We have $\gamma^{T} x^{*}=\gamma^{T} x(0)$ due to invariance of $\gamma^{T} x(t)$. But $x^{*}=\alpha \mathbf{1}$, thus we obtain

$$
\left(\sum_{i} \gamma_{i}\right) \alpha=\gamma^{T} x(0)
$$

and the result follows.

The following result shows that if one of the agents uses a relatively small update rate (or step-size), i.e. $\gamma_{i^{*}} \gg \gamma_{i}$ for all $i \neq i^{*}$. Then, the value of all nodes converges to the value of $x_{i}^{*}$. In other words, the agent $i^{*}$ plays the role of a leader in leader-follower type architecture.

Corollary 3. (multi-rate integrators) Consider a network of multi-rate integrators with the node dynamics

$$
\gamma_{i} \dot{x}_{i}=u_{i}, \quad \gamma_{i}>0, \forall i \in \mathcal{I}
$$

Assume each node applies Protocol (A1). Then, an agreement is globally asymptotically reached and the decision value of the group is

$$
\alpha=\frac{\sum_{i} \gamma_{i} x_{i}(0)}{\sum_{i} \gamma_{i}}
$$

Proof. The dynamics of the network evolves according to

$$
D \dot{x}=-L x
$$

where $D=\operatorname{diag}(\gamma)$ is a diagonal matrix with the $i$ th diagonal element that is equal to $\gamma_{i}>0$. The last equation can be rewritten as

$$
\dot{x}=-\tilde{L} x
$$

where $\tilde{L}=D^{-1} L=\operatorname{diag}\left(1 / \gamma_{1}, \ldots, 1 / \gamma_{n}\right) L$ is a valid Laplacian matrix for a digraph $\tilde{G}$ with adjacency matrix $\tilde{A}=D^{-1} \mathcal{A}$ (i.e. the weights of the edges leaving node $i$ are divided by $\gamma_{i}$ ). Clearly, $\gamma$ is a vector with positive elements that is the left eigenvector of $\tilde{L}$ and based on Corollary 2 the decision value is in the weighted average of $x_{i}(0)$ 's with weights specified by $\gamma$.

Remark 6. The discrete-time model and attitude alignment protocol discussed in Jadbabaie et al. [13] correspond to the first-order Euler approximation of equation (20) with protocol (A1) and the special choice of $\gamma_{i}=\operatorname{deg}_{\text {out }}\left(v_{i}\right)+1$ in Corollary 3. In [6], a Laplacian matrix is defined as $I-D \mathcal{A}$ which in the context of this paper is equivalent to a multi-rate network of integrators with $\gamma_{i}=\operatorname{deg}_{\text {out }}\left(v_{i}\right) \geq 0$. The singularity of $D$ that is caused by the choice of $\gamma_{i}=\operatorname{deg}_{\text {out }}\left(v_{i}\right)$ is avoided in [13] by properly adding a positive constant (e.g. 1) to $\operatorname{deg}_{\text {out }}\left(v_{i}\right)$. 
Theorem 6. Let $G=(\mathcal{V}, \mathcal{E}, \mathcal{A})$ be a digraph with an adjacency matrix $\mathcal{A}=\left[a_{i j}\right]$. Then, all the following statements are equivalent:

i) $G$ is balanced,

ii) $w_{l}=\mathbf{1}$ is the left eigenvector of the Laplacian of $G$ associates with the zero eigenvalue, i.e. $\mathbf{1}^{T} L=0$.

iii) $\sum_{i=1}^{n} u_{i}=0, \forall x \in \mathbb{R}^{n}$ with $u_{i}=\sum_{j \in N_{i}} a_{i j}\left(x_{j}-x_{i}\right)$.

Proof. We show $i) \Longleftrightarrow i i)$ and $i i) \Longleftrightarrow i i i)$.

Proof of $i) \Longleftrightarrow i i)$ : We have $\Delta_{i i}=\operatorname{deg}_{\text {out }}\left(v_{i}\right)$ and $\operatorname{deg}_{i n}\left(v_{i}\right)=\sum_{j, j \neq i} a_{j i}$, thus the $i$ th column sum of $L$ is equal to

$$
\sum_{i} l_{j i}=\sum_{i, j \neq i} l_{j i}+l_{i i}=-\operatorname{deg}_{i n}\left(v_{i}\right)+\operatorname{deg}_{\text {out }}\left(v_{i}\right)=0 \Longleftrightarrow \text { node } v_{i} \text { of } G \text { is balanced }
$$

Noting that the $i$ column sum of $L$ is the same as the $i$ th element of the row vector $\mathbf{1}^{T} L$, one concludes that $\mathbf{1}^{T} L=0$ iff all the nodes of $G$ are balanced, i.e. $G$ is balanced.

Proof of $i i) \Longleftrightarrow i i i)$ : Since $u=-L x,\left(\sum_{i} u_{i}=0, \forall x\right) \Leftrightarrow\left(\mathbf{1}^{T} u=-\left(\mathbf{1}^{T} L\right) x=\right.$ $0, \forall x) \Longleftrightarrow \mathbf{1}^{T} L=0$.

Remark 7. Notice that in Theorem 6, the graph $G$ does not need to be connected.

\subsection{Performance of Group Agreement and Mirror Graphs}

In this section, we discuss performance issues of Protocol (A1) with balanced graphs. An important consequence of Theorem 6 is that for networks with balanced information flow, $\alpha=$ Ave $(x)$ is an invariant quantity. This is certainly not true for an arbitrary digraph. The invariance of $\operatorname{Ave}(x)$ allows decomposition of $x$ according to the following equation:

$$
x=\alpha \mathbf{1}+\delta
$$

where $\alpha=\operatorname{Ave}(x)$ and $\delta \in \mathbb{R}^{n}$ satisfies $\sum_{i} \delta_{i}=0$. We refer to $\delta$ as the (group) disagreement vector. The vector $\delta$ is orthogonal to 1 and belongs to an $(n-1)$-dimensional subspace called the disagreement eigenspace of $L$ provided that $G$ is strongly connected. Moreover, $\delta$ evolves according to the (group) disagreement dynamics given by

$$
\dot{\delta}=-L \delta
$$

Define the Laplacian disagreement function of a digraph $G$ as

$$
\Phi_{G}(x)=x^{T} L x
$$

with $L=\mathcal{L}(G)$. For digraphs, $\Phi_{G}(x)$ can be negative (e.g. the Laplacian of a digraph with two nodes and a single edge 12 is not positive semidefinite). 
It turns out that a useful property of balanced digraphs is that their Laplacian disagreement function is positive semidefinite. In addition, for any balanced digraph $G$, there exists an undirected graph that has the same Laplacian disagreement function as $G$. In the following, we formally define this induced undirected graph.

Definition 2. (mirror graph/operation) Let $G=(\mathcal{V}, \mathcal{E}, \mathcal{A})$ be weighted digraph. Let $\tilde{\mathcal{E}}$ be the set of reverse edges of $G$ obtained by reversing the order of nodes of all the pairs in $\mathcal{E}$. The mirror of $G$ denoted by $\hat{G}=\mathcal{M}(G)$ is an undirected graph in the form $\hat{G}=(\mathcal{V}, \hat{\mathcal{E}}, \hat{\mathcal{A}})$ with the same set of nodes as $G$, the set of edges $\hat{\mathcal{E}}=\mathcal{E} \cup \tilde{\mathcal{E}}$, and the symmetric adjacency matrix $\hat{\mathcal{A}}=\left[\hat{a}_{i j}\right]$ with elements

$$
\hat{a}_{i j}=\hat{a}_{j i}=\frac{a_{i j}+a_{j i}}{2} \geq 0
$$

The following result shows that the operations of $\mathcal{L}$ and $S y m$ on a weighted adjacency matrix $\mathcal{A}$ commute.

Theorem 7. Let $G$ be a digraph with adjacency matrix $\mathcal{A}=\operatorname{adj}(G)$ and Laplacian $L=\mathcal{L}(G)$. Then $L_{s}=\operatorname{Sym}(L)=\left(L+L^{T}\right) / 2$ is a valid Laplacian matrix for $\hat{G}=\mathcal{M}(G)$ if and only if $G$ is balanced, i.e. the following diagram commutes iff $G$ is balanced

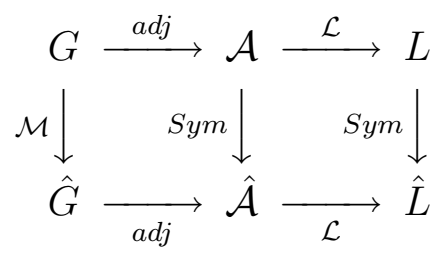

Moreover, if $G$ is balanced, the Laplacian disagreement functions of $G$ and $\hat{G}$ are equal.

Proof. We know that $G$ is balanced iff $\mathbf{1}^{T} L=0$. Since $L \mathbf{1}=0$, we have $\mathbf{1}^{T} L=0 \Longleftrightarrow$ $\frac{1}{2}\left(L+L^{T}\right) \mathbf{1}=0$. Thus, $G$ is balanced iff $L_{s}$ has a right eigenvector of $\mathbf{1}$ associated with $\lambda=0$, i.e. $L_{s}$ is a valid Laplacian matrix. Now, we prove that $L_{s}=\mathcal{L}(\hat{G})$. For doing so, let us calculate $\hat{\Delta}$ element-wise, we get

$$
\hat{\Delta}_{i i}=\sum_{j} \frac{a_{i j}+a_{j i}}{2}=\frac{1}{2}\left(\operatorname{deg}_{\text {out }}\left(v_{i}\right)+\operatorname{deg}_{\text {in }}\left(v_{i}\right)\right)=\operatorname{deg}_{\text {out }}\left(v_{i}\right)=\Delta_{i i}
$$

Thus, $\hat{\Delta}=\Delta$. On the other hand, we have

$$
L_{s}=\frac{1}{2}\left(L+L^{T}\right)=\Delta-\frac{A+A^{T}}{2}=\hat{\Delta}-\hat{A}=\hat{L}=\mathcal{L}(\hat{G})
$$

The last part simply follows from the fact that $\hat{L}$ is equal to the symmetric part of $L$ and $x^{T}\left(L-L^{T}\right) x \equiv 0$. 
Notation. For simplicity of notation, in the context of algebraic graph theory, $\lambda_{k}(G)$ is used to denote $\lambda_{k}(\mathcal{L}(G))$.

Now, we are ready to present our main result on performance of the Protocol (A1) in terms of the speed of reaching a consensus as a group.

Theorem 8. (performance of agreement) Consider a network of integrators with a directed information flow $G$ that is balanced and strongly connected. Then, given Protocol (A1), the following statements hold:

i) the group disagreement (vector) $\delta$ as the solution of the disagreement dynamics in (23) globally asymptotically vanishes with a speed that is equal to $\kappa=\lambda_{2}(\hat{G})$ (or the Fiedler eigenvalue of the mirror graph of $G$ ), i.e.

$$
\|\delta(t)\| \leq\|\delta(0)\| \exp (-\kappa t)
$$

ii) the following smooth, positive definite, and proper function

$$
V(\delta)=\frac{1}{2}\|\delta\|^{2}
$$

is a valid Lyapunov function for the disagreement dynamics.

Proof. We have

$$
\dot{V}=-\delta^{T} L \delta=-\delta^{T} L_{s} \delta=-\delta^{T} \hat{L} \delta \leq-\lambda_{2}(\hat{G})\|\delta\|^{2}=-2 \kappa V(\delta)<0, \forall \delta \neq 0
$$

This proves that $V(\delta)$ is a valid Lyapunov function for the group disagreement dynamics. Moreover, $\delta(t)$ vanishes globally exponentially fast with a speed of $\kappa$ as $t \rightarrow+\infty$.. The fact that $L_{s}=\hat{L}$ is a valid Laplacian matrix for an undirected graph (i.e. mirror of $G$ ) follows from Theorem 7 and the inequality

$$
\delta^{T} \hat{L} \delta \geq \lambda_{2}(\hat{G})\|\delta\|^{2}, \quad \forall \delta: \mathbf{1}^{T} \delta=0
$$

which is due to equation (14).

A well-known observation regarding the Fiedler eigenvalue of an undirected graph is that for dense graphs $\lambda_{2}$ is relatively large and for sparse graphs $\lambda_{2}$ is relatively small [11] (this is why $\lambda_{2}$ is called the algebraic connectivity). According to this observation, from Theorem 8 , one can conclude that a network with dense interconnections solves an agreement problem faster than a connected but sparse network. This is consistent with common sense regarding agreement in a group. As a special case, a cycle of length $n$ that creates a balanced digraph on $n$ nodes solves an agreement problem. However, this is a relatively slow way to solve such a consensus problem. 


\subsection{Consensus in Networks with Switching Topology}

Consider a network of mobile agents that communicate with each other and need to agree upon a certain objective of interest or perform synchronization. Since, the nodes of the network are moving, it is not hard to imagine that some of the existing communication links can fail simply due to the existence of an obstacle between two agents. The opposite situation can arise where new links between nearby agents are created because the agents come to an effective range of detection with respect to each other. In other words, in the graph $G$ representing the information flow of the network, certain edges can be added or removed from $G$. Here, we are interested to investigate that in case of a network with switching topology whether it is still possible to reach a consensus or not.

Consider a hybrid system with a continuous-state $x \in \mathbb{R}^{n}$ and a discrete-state $G$ that belongs to a finite set of digraphs

$$
\Gamma_{n}=\{G: G \text { is a digraph of order } n \text { that is strongly connected and balanced }\}
$$

that can be analytically expressed in the form

$$
\Gamma_{n}=\left\{G=(\mathcal{V}, \mathcal{E}, \mathcal{A}): \operatorname{rank}(\mathcal{L}(G))=n-1, \mathbf{1}^{T} \mathcal{L}(G)=0\right\}
$$

Given the node dynamics and protocol, the continuous-state of the system evolves according to the following dynamics

$$
\dot{x}(t)=-\mathcal{L}\left(G_{k}\right) x(t), \quad k=s(t), G_{k} \in \Gamma_{n}
$$

where $s(t): \mathbb{R}_{\geq 0} \rightarrow \mathcal{I}_{\Gamma_{n}}$ is a switching signal and $\mathcal{I}_{\Gamma_{n}} \subset \mathbb{N}$ is the index set associated with the elements of $\Gamma_{n}$. Clearly, $\Gamma_{n}$ is a finite set, because either a digraph has no edges or it is a complete graph with $n(n-1)$ directed edges.

The key in solving the agreement problem for mobile networks with switching topology is a basic property of the Lyapunov function in (28) and the properties of balanced graphs. Note that the function $V(\delta)=\frac{1}{2}\|\delta\|^{2}$ does not depend on $G$ or $L=\mathcal{L}(G)$. This property of $V(\delta)$ makes it an appropriate candidate as a common Lyapunov function for stability analysis of the switching system (32).

Theorem 9. For any arbitrary switching signal $s(\cdot)$, the solution of the switching system (32) globally asymptotically converges to Ave $(x(0))$ (i.e. average-consensus is reached). Moreover, the following smooth, positive definite, and proper function

$$
V(\delta)=\frac{1}{2}\|\delta\|^{2}
$$

is a valid common Lyapunov function for the disagreement dynamics given by

$$
\dot{\delta}(t)=-\mathcal{L}\left(G_{k}\right) \delta(t), \quad k=s(t), G_{k} \in \Gamma_{n} .
$$

Furthermore, the disagreement vector $\delta$ vanishes exponentially fast with the least rate of

$$
\left.\kappa^{*}=\min _{G \in \Gamma_{n}} \lambda_{2}(\mathcal{L}(\hat{G}))\right)
$$

In other words, $\|\delta(t)\| \leq\|\delta(0)\| \exp \left(-\kappa^{*} t\right)$. 
Proof. Due the fact that $G_{k}$ is balanced for all $k$ and $u=-\mathcal{L}\left(G_{k}\right) x$, we have $\mathbf{1}^{T} u=$ $-\left(\mathbf{1}^{T} \mathcal{L}\left(G_{k}\right)\right) x \equiv 0$. Thus, $\alpha=\operatorname{Ave}(x)$ is an invariant quantity which allows us to decompose $x$ as $x=\alpha \mathbf{1}+\delta$. Therefore, the disagreement switching system induced by (32) takes the form (34). Calculating $\dot{V}$, we get

$$
\dot{V}=-\delta^{T} \mathcal{L}\left(G_{k}\right) \delta=-\delta^{T} \mathcal{L}\left(\hat{G}_{k}\right) \delta \leq-\lambda_{2}\left(\mathcal{L}\left(\hat{G}_{k}\right)\right)\|\delta\|^{2} \leq-\kappa^{*}\|\delta\|^{2}=-2 \kappa^{*} V(\delta)<0, \forall \delta \neq 0
$$

This guarantees that $V(\delta)$ is a valid common Lyapunov function for the disagreement switching system (34). Moreover, we have

$$
V(\delta(t)) \leq V(\delta(0)) \exp \left(-2 \kappa^{*} t\right) \Rightarrow\|\delta(t)\| \leq\|\delta(0)\| \exp \left(-\kappa^{*} t\right)
$$

and the disagreement vector $\delta(t)$ globally exponentially vanishes with a speed of $\kappa^{*}>0$ as $t \rightarrow+\infty$. Finally, the minimum in (35) always exists and is achieved because $\Gamma_{n}$ is a finite set.

\section{Networks with Communication Time-Delays}

Consider a network of continuous-time integrators with a fixed topology $G=(\mathcal{V}, \mathcal{E}, \mathcal{A})$ in which the state of node $i$ passes through a communication channel (or link) $i j$ with timedelay $\tau_{i j}>0$ before getting to node $j$. The transfer function of link $i j$ can be expressed as

$$
h_{i j}(s)=e^{-\tau_{i j} s}
$$

in the Laplace domain. Applying the time-delayed linear protocol (A2), the network dynamics can be written as

$$
\dot{x}_{i}(t)=\sum_{j \in N_{i}} a_{i j}\left[x_{j}\left(t-\tau_{i j}\right)-x_{i}\left(t-\tau_{i j}\right)\right] .
$$

After taking the Laplace transform of both sides of equation (37), we get

$$
s X_{i}(s)-x_{i}(0)=\sum_{j \in N_{i}} a_{i j} h_{i j}(s)\left(X_{j}(s)-X_{i}(s)\right)
$$

where $X_{i}(s)$ denotes the Laplace transform of $x_{i}(t)$ for all $i \in \mathcal{I}$. The last set of equations can be rewritten in a compact form as

$$
X(s)=(s+L(s))^{-1} x(0)
$$

where $L(s)$ is the Laplacian matrix of a graph with adjacency matrix $\mathcal{A}(s)=\left[a_{i j} h_{i j}(s)\right]$. In general, any filtering effect of channel $i j$ can be incorporated in the link transfer function $h_{i j}(s)$. The convergence analysis of protocol (A2) for a network of integrator agents with communication time-delays reduces to stability analysis for a MIMO transfer function

$$
G(s)=(s I+L(s))^{-1} .
$$


To gain further insight in the relation between the graph Laplacian and the convergence properties of the consensus protocol (A2), we focus on the simplest possible case where the time-delays in all channels are equal to $\tau>0$ in a network with an undirected and fixed topology $G$. Immediately, it follows that $\sum_{i} u_{i} \equiv 0$ and thus $\alpha=\operatorname{Ave}(x(t))$ is an invariant quantity. In addition, we have

$$
L(s)=e^{-\tau s} L
$$

where $L=\mathcal{L}(G)$. Here is our main result for average-consensus in a network with communication time-delays [20]:

Theorem 10. Consider a network of integrator agents with equal communication time-delay $\tau>0$ in all links. Assume the information flow $G$ of the network is undirected and connected. Then, protocol (A2) with $\tau_{i j}=\tau$ globally asymptotically solves average-consensus problem if and only if either of the following two equivalent conditions are satisfied:

i) $\tau \in\left(0, \tau^{*}\right)$ with $\tau^{*}=\frac{\pi}{2 \lambda_{n}}, \lambda_{n}=\lambda_{\max }(L)$.

ii) The Nyquist plot of $\Gamma(s)=e^{-\tau s} / s$ has a zero encirclement around $-1 / \lambda_{k}, \forall k>1$.

Moreover, for $\tau=\tau^{*}$ the system has a globally asymptotically stable oscillatory solution with frequency $\omega=\lambda_{n}$.

Proof. See Section A.2 in the Appendix.

Based on part i) of Theorem 10, one concludes that the upper bound on the admissible channel time-delay in the network is inversely proportional to $\lambda_{n}$, i.e. the largest eigenvalue of the Laplacian of the information flow. From Geršgorin theorem, we know that $\lambda_{n} \leq 2 d_{\max }(G)$ where $d_{\text {max }}(G)$ is the maximum out-degree of the nodes of $G$. Therefore, a sufficient condition for convergence of protocol (A2) is

$$
\tau \leq \frac{\pi}{4 d_{\max }(G)}
$$

This means that networks with nodes that have relatively high out-degrees cannot tolerate relatively high communication time-delays. On the other hand, let $\tilde{\mathcal{A}}=k \mathcal{A}$ with $k>0$ be the adjacency matrix of $\tilde{G}$. Let $\tilde{L}=\mathcal{L}(\tilde{G})$ and notice that $\lambda_{n}(\tilde{L})=k \lambda_{n}(L)$. Thus, for any arbitrary delay $\tau>0$, there exists a sufficiently small $k>0$ such that $\tau<\pi /\left(2 k \lambda_{n}\right)$. As a result, by scaling the weights of the digraph, any arbitrary time-delay can be tolerated. The trade-off is that the negotiation speed degrades by a factor of $1 / k>0$. In other words, there is trade-off between robustness to time-delays and speed of convergence (or performance) of the agreement algorithm.

\section{Max-Consensus and Leader Determination}

In this section, we discuss the problem of max-consensus in a network of agents with a unique max-leader, i.e. a node $i^{*}=\arg \max _{i} x_{i}(0)$. The max-consensus problem can be described 
as follows. Each agent has the following discrete-time model

$$
x_{i}(k+1)=\max \left(x_{i}(k), u_{i}(k)\right)
$$

and is called a max-agent. The dynamics of a max-agent can be equivalently expressed as a nonlinear discrete-time system

$$
x_{i}(k+1)=\frac{1}{2}\left(x_{i}(k)+u_{i}(k)+\left|x_{i}(k)-u_{i}(k)\right|\right)
$$

The value of all nodes in the $\operatorname{digraph} G=(\mathcal{V}, \mathcal{E})$ has to iteratively converge to the value of the max-leader. In addition, each node in a distributed way has to determine whether it is the max-leader or not. One of the applications of the max-consensus problem is to determine a leader (or coordinator) in a group with superiority in terms of a particular quantity of interest (e.g. index number, cost, height, and so on) that has to perform or coordinate a task.

We use the following protocol to solve the max-consensus problem:

$$
u_{i}(k)=\max _{j \in N_{i}} x_{j}
$$

Remark 8. Apparently, the min-consensus problem can be defined and solved in a similar way to the max-consensus problem and will not be discussed here.

Notice that the value of each max-agent is non-decreasing, i.e. $x_{i}(k+1) \geq x_{i}(k)$ for all $i \in \mathcal{I}$. To keep track of whether a node is the max-leader or not, we augment the state each agent with a Boolean variable $f_{i}(k) \in\{0,1\}$ called the max-flag of node $i$. The max-flag evolves according to the following rule (or state feedback)

$$
f_{i}(k+1)=K\left(f_{i}(k), x_{i}(k), u_{i}(k)\right):= \begin{cases}f_{i}(k) & x_{i}(k+1)=x_{i}(k) \\ \bar{f}_{i}(k) & x_{i}(k+1)>x_{i}(k)\end{cases}
$$

where the bar operation negates a Boolean variable. Initially, all nodes assume that they are the max leader, or $f_{i}(0)=1$ for all $i$. If the value of each node increases compared to its initial value, then it realizes that it is no more a max-leader.

Theorem 11. Consider a network of max-agents with the following dynamics

$$
\left\{\begin{aligned}
x_{i}(k+1) & =\max \left(x_{i}(k), u_{i}(k)\right) \\
f_{i}(k+1) & =K\left(f_{i}(k), x_{i}(k), u_{i}(k)\right)
\end{aligned}\right.
$$

where the map $K:\{0,1\} \times \mathbb{R} \times \mathbb{R} \rightarrow\{0,1\}$ is defined in $(43)$. Assume $G=(\mathcal{V}, \mathcal{E})$ is a strongly connected digraph. Then, protocol (A4) solves the max-consensus problem in finite number of iterations $l \leq n-1$, i.e. the value of all nodes converges to the value of the max-leader and the max-flag of all nodes but the max-leader converges to zero in $O(n)$ time. 


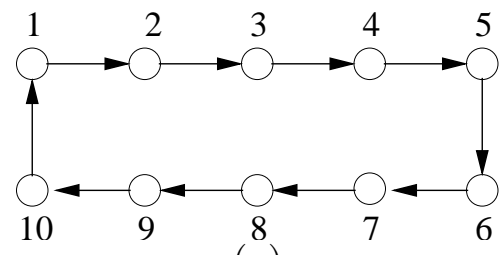

(a)

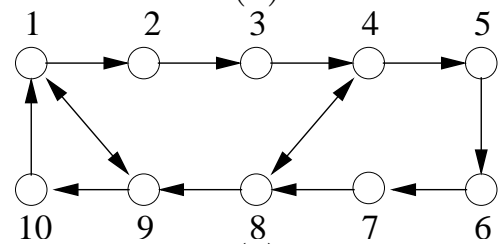

(c)

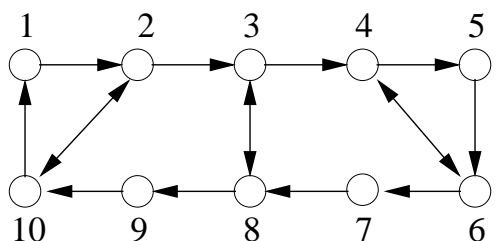

(b)

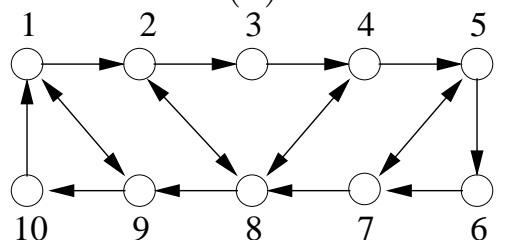

$(\mathrm{d})$

Figure 4: For examples of balanced and strongly connected digraphs: (a) $G_{a}$, (b) $G_{b}$, (c) $G_{c}$, and (d) $G_{d}$ satisfying.

Proof. Let $l$ be the maximum length of the shortest path connecting each node of the digraph to the max-leader. Since $G$ is strongly connected $l \leq n-1$. Observe that the value of the max-leader $\alpha=x_{i}(k)$ remains invariant in time, because the value of all other nodes are smaller than the value of the max leader and from $(43), f_{i^{*}}(k)=f_{i^{*}}(0)=1$ for all $k>0$. Similar to the line of proof of Theorem 1 , let $J^{(k)}$ denote a cluster of nodes that are the $k$ th neighbors of the max-leader $i^{*}$ (for the definition of $J^{(k)}$, see equation (46) in the Appendix). Given protocol (A4), it is easy to see that the value of all the $k$ th neighbors of $i^{*}$ becomes equal to $\alpha$ and the max-flag of all nodes in $J^{(k)} \backslash\left\{i^{*}\right\}$ becomes zero after $k$ iterations. On the other hand, by definition of $l \leq n-1, J^{(l)}=\mathcal{I}$ and the result follows.

\section{Simulation Results}

Figure 4 shows four different networks each with $n=10$ nodes that are all strongly connected and balanced. The weights associated with all the edges are 1. For the following initial node values satisfying $\operatorname{Ave}(x(0))=0$

$x(0)=(-10.2999,0.2575,-4.4997,3.6258,3.0922,9.0156,3.5099,-2.6645,2.4552,-4.4921)^{T}$

we have plotted the state trajectories and the disagreement function $\|\delta\|^{2}$ associated with these four digraphs in Figure 5. It is clear that as the number of the edges of the graph increase, algebraic connectivity (or $\lambda_{2}$ ) increases, and the settling time of the trajectory of the node values decreases. The case of a directed cycle of length $n=10$, or $G_{a}$, has the largest over-shoot. In all four cases, an agreement is asymptotically reached and the performance is improved as a function of $\lambda_{2}\left(\hat{G}_{k}\right)$ for $k \in\{a, b, c, d\}$.

In Figure 6(a), a finite automaton is shown with the set of states $\left\{G_{a}, G_{b}, G_{c}, G_{d}\right\}$ representing the discrete-states of a network with switching topology as a hybrid system. The hybrid system starts at the discrete-state $G_{b}$ and switches every $T=1$ second to the next 

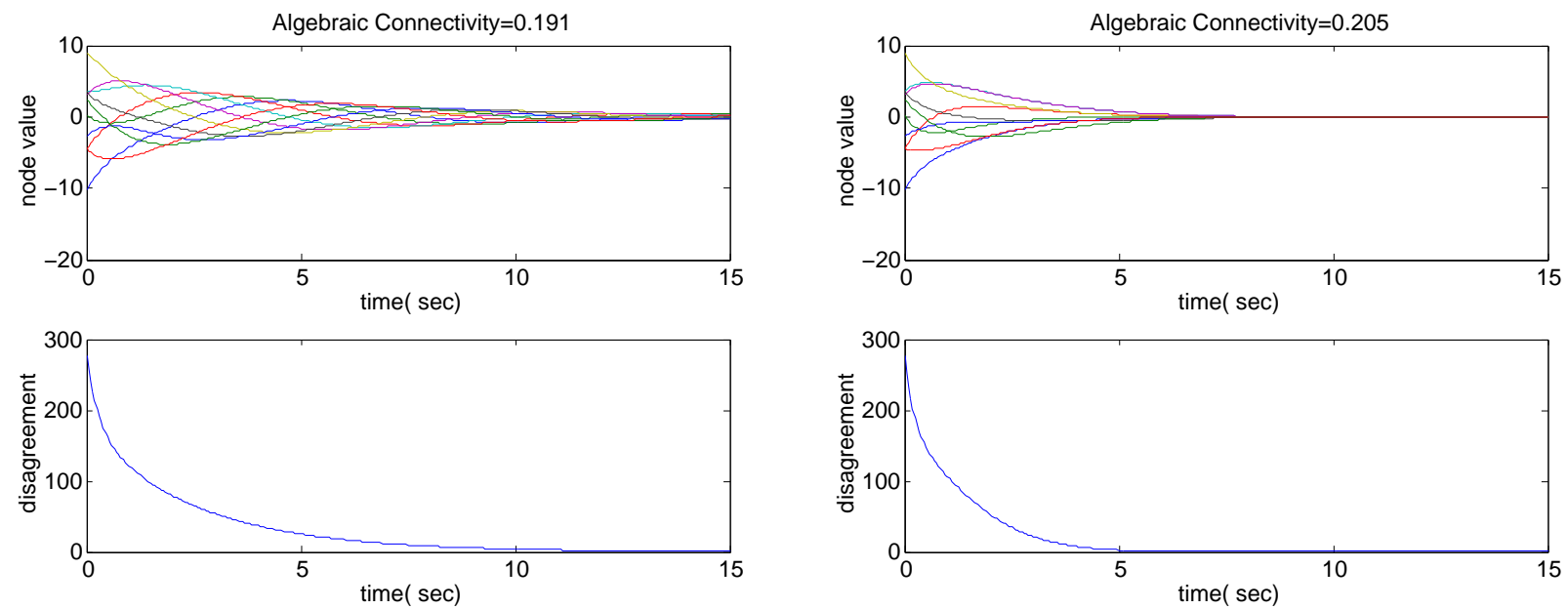

(a)

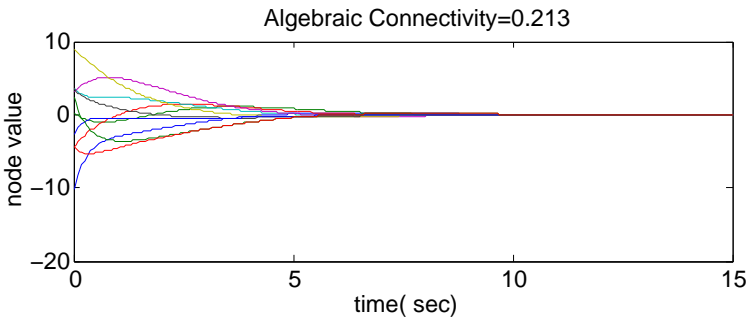

(b)
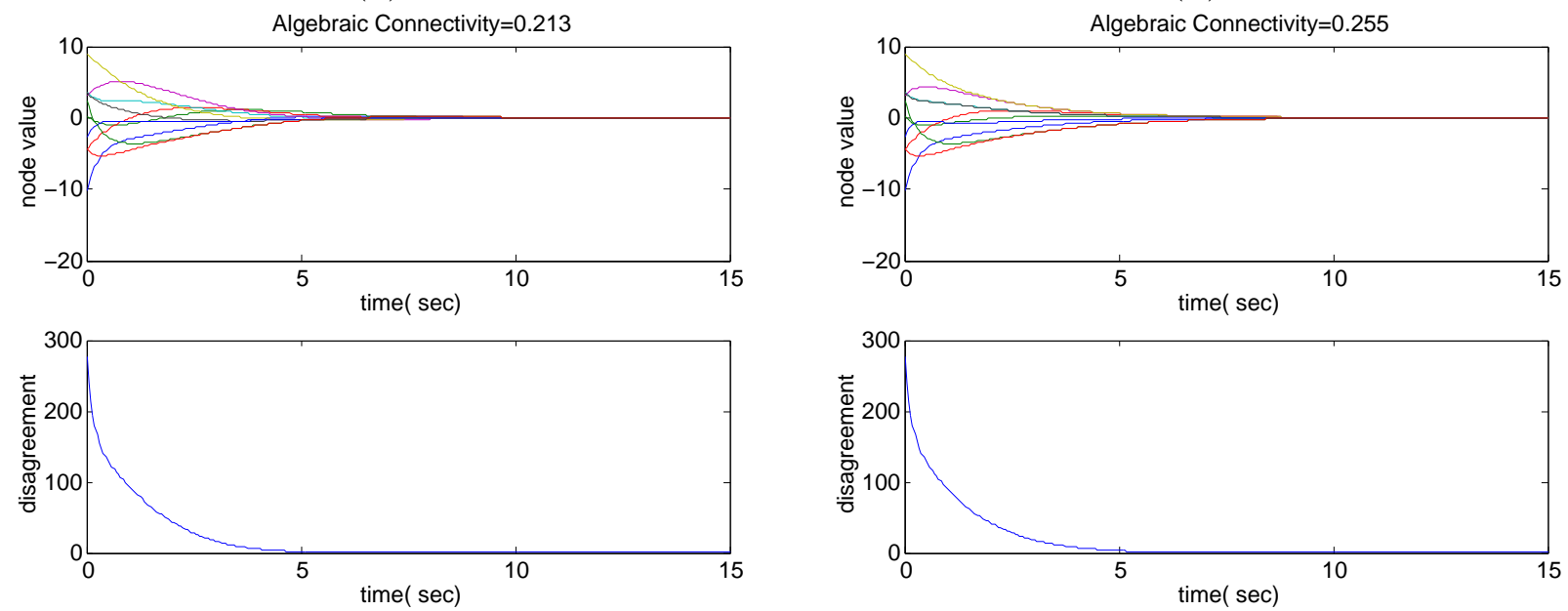

(c)

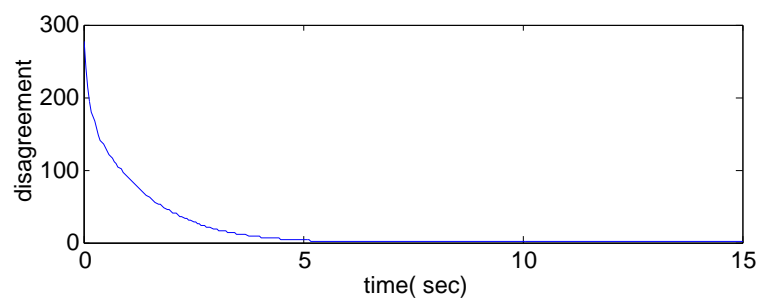

(d)

Figure 5: For examples of balanced and strongly connected digraphs: (a) $G_{a}$, (b) $G_{b}$, (c) $G_{c}$, and (d) $G_{d}$ satisfying.

state according to the state machine in Figure 6(a). The continuous-time state trajectories and the group disagreement (i.e. $\|\delta\|^{2}$ ) of the network are shown in Figure 6(b). Clearly, the group disagreement is monotonically decreasing. One can observe that an average-consensus is reached asymptotically. Moreover, the group disagreement vanishes exponentially fast.

Next, we present simulation results for average-consensus problem with communication time-delays for a network with information flow shown in Figure 7. Figure 8 shows the state trajectories of $n=10$ nodes for a network with communication time-delay $\tau$ for $\tau=$ $0,0.5 \tau_{\max }, \tau_{\max }=\pi / 2 \lambda_{\max }\left(G_{a}\right)=0.266$ for a zero-mean random set of initial conditions. Clearly, the agreement is achieved for the cases with $\tau<\tau_{\max }$ in Figures 8(a), (b), and (c). For the case with $\tau=\tau_{\max }$, synchronous oscillations are demonstrated in Figure 8(d). A third-order Pade approximation is used to model the time-delay as a finite-order LTI system. 


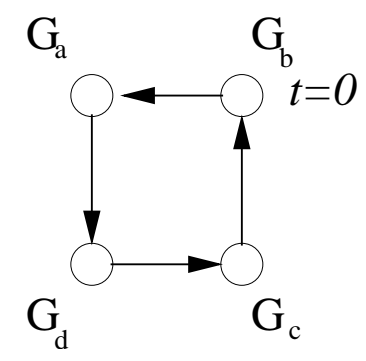

(a)
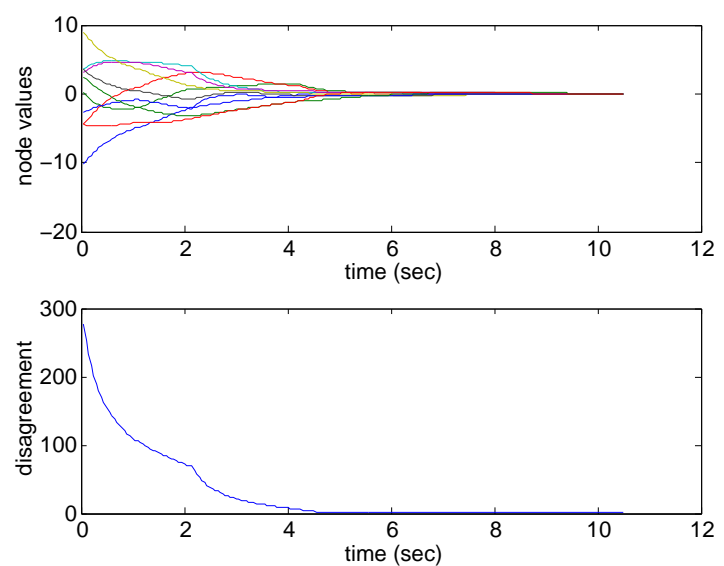

(b)

Figure 6: (a) A finite automaton with four states representing the discrete-states of a network with variable topology and (b) trajectory of the node values and the group disagreement for a network with a switching information flow.

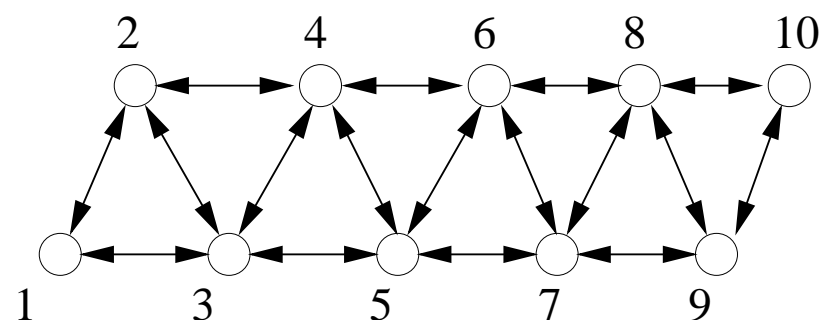

Figure 7: Undirected graph $G_{e}$ used for consensus with communication time-delays

\section{Conclusions}

We provided convergence analysis of consensus protocol for a network of integrators with a directed information flow and fixed or switching topology. Our analysis relies on several tools from algebraic graph theory and matrix theory. We established a connection between the performance of the linear consensus protocol and the Fiedler eigenvalue of graph Laplacian of the mirror graph. A simple disagreement function was introduced as a Lyapunov function for the group disagreement dynamics. This was later used to provide a common Lyapunov function that allowed convergence analysis of an agreement protocol for a network with switching topology. A commutative diagram was given that shows the operations of taking Laplacian and symmetric part of a matrix commute for weighted adjacency matrices of balanced graphs. Balanced graphs turned out to be instrumental in solving average-consensus problems.

For networks with undirected graphs, we gave sufficient and necessary conditions for consensus in networks with communication time-delays. It was shown that there is a tradeoff between robustness to time-delays and the speed of convergence of a linear consensus 


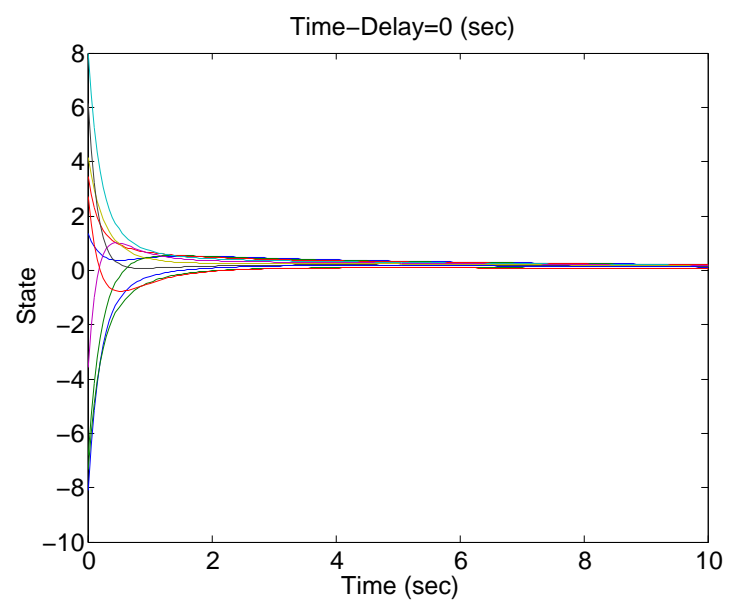

(a)

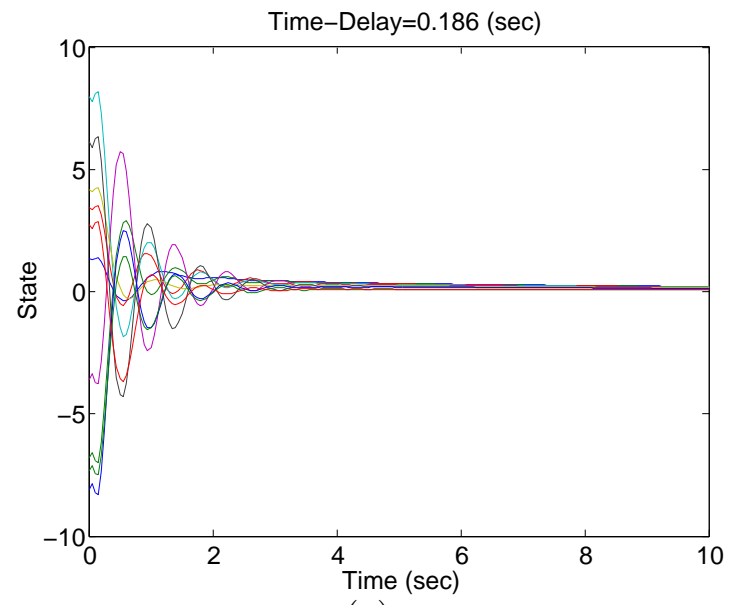

(c)

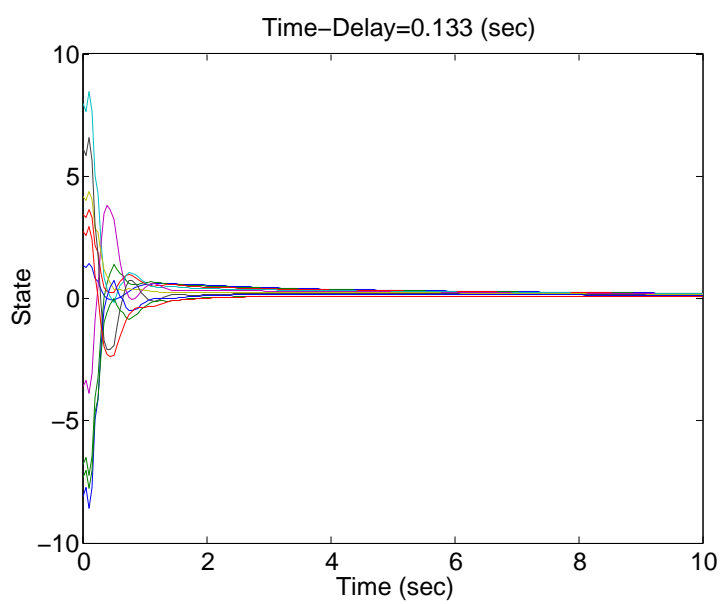

(b)

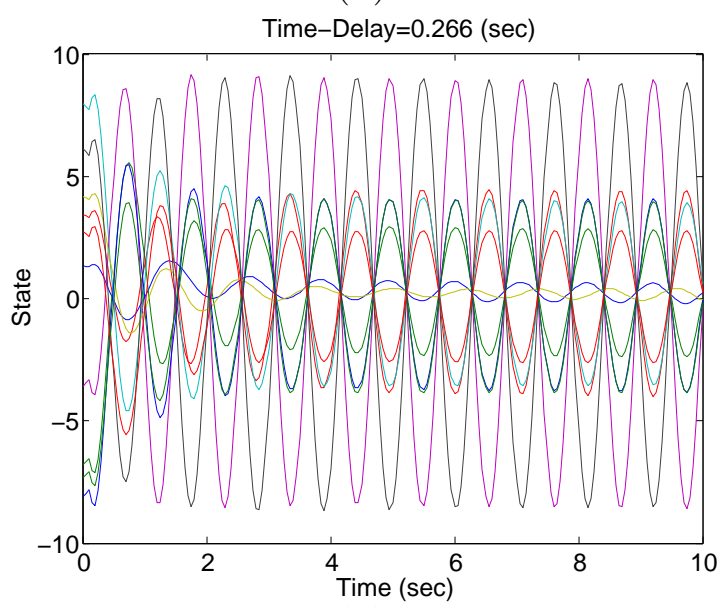

(d)

Figure 8: Consensus problem with communication time-delay on $G_{b}$ in Figure 7: (a) $\tau=0$, (b) $\tau=0.5 \tau_{\max },(\mathrm{c}) \tau=0.7 \tau_{\max }$, and $(\mathrm{d}) \tau=\tau_{\max }$.

protocol.

To determine a superior or leader in a group of agents, we introduced max-agents that have a very simple discrete-time model and gave a protocol that solves the max-consensus problem in a distributed way after a maximum of $n-1$ iterations ( $n$ is the number of agents).

We presented extensive simulation results that demonstrate the effectiveness of our theoretical results.

The consensus problems for discrete-time approximation of integrator agents relies on the theory of nonnegative matrices and will be presented in an upcoming paper. 


\section{Acknowledgments}

This research is supported in part by AFOSR under the grant F49620-01-1-0361 and by DARPA under the grant F33615-98-C-3613.

\section{A Appendix}

This section contains the proofs of some of the theorems of the paper.

\section{A.1 Proof of Theorem 1}

Proof. Define $\phi_{i j}(z)=a_{i j} z$ for all $i j \in \mathcal{E}$. It is trivial that if $x_{i}=x_{j}$ for all $i j \in \mathcal{E}$, then $u=0$. Thus, we prove the converse: $u=0$ implies that all nodes are in agreement. If the values of all nodes are equal, the result follows. Thus, assume there exists a node $i^{*}$, called max-leader, such that $x_{i^{*}} \geq x_{j}$ for all $j \neq i^{*}$, i.e. $i^{*}=\arg \max _{j \in \mathcal{I}} x_{j}$ (if $i^{*}$ is not unique, choose one arbitrarily).

Define the initial cluster $J^{(0)}=\left\{i^{*}\right\}$ and denote the indices of all the first-neighbors of $i^{*}$ by $J^{(1)}=N_{i^{*}}$. Then, $u_{i^{*}}=0$ implies that

$$
\sum_{j \in N_{i^{*}}} \phi_{i^{*} j}\left(x_{j}-x_{i^{*}}\right)=0
$$

Since $x_{j} \leq x_{i^{*}}$ for all $j \in N_{i^{*}}$ and $\phi_{i j}(z) \leq 0$ for $z \leq 0$ (i.e. all weights are nonnegative), we get $x_{i^{*}}=x_{j}$ for all the first-neighbors $j \in J^{(1)}$, (i.e. the max-leader and all of its firsneighbors are in agreement). Next, we define the $k$ th-neighbors of $i^{*}$ and show that the max-leader is in agreement with all of its $k$ th-neighbors for $k=1, \ldots, n-1$. The set of $k$ th-neighbors of $i^{*}$ is defined by the following recursive equation

$$
J^{(k)}=J^{(k-1)} \cup N_{J^{(k-1)}}, \quad k \geq 1, J^{(0)}=\left\{i^{*}\right\}
$$

where $N_{J}$ denotes the set of neighbors of cluster $J \subseteq \mathcal{I}$ (see equation (1)). By definition, $\left\{i^{*}\right\} \subset J^{(k)} \subseteq \mathcal{I}$ for $k \geq 1$ and $J^{(k)}$ is a monotonically increasing sequence of clusters (in terms of inclusion).

Notice that in a strongly connected digraph, the maximum length of the minimum path connecting any node $j \neq i^{*}$ to node $i^{*}$ is $n-1$. Thus, $J^{(n-1)}=\mathcal{I}$. By induction, we prove that all the nodes in $J^{(k)}$ are in agreement for $k \geq 1$. The statement holds for $k=1$ (i.e. the set of first-neighbors of the max-leader). Assume all the nodes in $J^{(k)}$ are in agreement with $i^{*}$, we show that all the nodes in $J^{(k+1)}$ are in agreement with $i^{*}$ as well. It is sufficient to show this for an arbitrary node $i \in J^{(k)}$ with $N_{i} \cap\left(J^{(k+1)} \backslash J^{(k)}\right) \neq \emptyset$ Otherwise, because in a strongly connected digraph $N_{i} \neq \emptyset$ for all $i$, we get $J^{(k+1)}=J^{(k)}$ and the statement holds. For node $i$, we have

$$
u_{i}=\sum_{j \in N_{i}} \phi_{i j}\left(x_{j}-x_{i}\right)=0
$$


But $N_{i}=\left(N_{i} \cap J^{(k)}\right) \cup\left(N_{i} \cap\left(\mathcal{I} \backslash J^{(k)}\right)\right)$ and $\mathcal{I} \backslash J^{(k)}=\mathcal{I} \backslash J^{(k+1)} \cup\left(J^{(k+1)} \backslash J^{(k)}\right)$. Keeping in mind that $J^{(k)} \subset$ equI for all $k$ and $J^{(k+1)}$ contains the set of first neighbors of node $i$, i.e. $N_{i} \subseteq J^{(k+1)}$, we have

$$
N_{i} \cap\left(\mathcal{I} \backslash J^{(k)}\right)=N_{i} \cap\left(J^{(k+1)} \backslash J^{(k)}\right)
$$

and

$$
u_{i}=\sum_{j \in N_{i} \cap J^{(k)}} \phi_{i j}\left(x_{j}-x_{i}\right)+\sum_{j \in N_{i} \cap\left(J^{(k+1)} \backslash J^{(k)}\right)} \phi_{i j}\left(x_{j}-x_{i}\right)=0
$$

The first sum is equal to zero because $x_{j}=x_{i}$ all nodes $j \in N_{i} \cap J^{(k)} \subseteq J^{(k)}$. Thus, the second sum must be zero. But $x_{i^{*}}=x_{i} \geq x_{j}$ for all $i \in J^{(k)}$ and $j \in \mathcal{I} \backslash J^{(k)}$ which implies all nodes in $N_{i} \cap\left(J^{(k+1)} \backslash J^{(k)}\right)$ are in agreement with $i^{*}$. This means that all nodes in the cluster

$$
\begin{aligned}
\cup_{i \in J^{(k)}} N_{i} \cap\left(J^{(k+1)} \backslash J^{(k)}\right) & =\left(\cup_{i \in J^{(k)}} N_{i}\right) \cap\left(J^{(k+1)} \backslash J^{(k)}\right) \\
& =J^{(k+1)} \cap\left(J^{(k+1)} \backslash J^{(k)}\right) \\
& =J^{(k+1)} \backslash J^{(k)}
\end{aligned}
$$

are in agreement with $i^{*}$, i.e. all the nodes in $J^{(k+1)}$ are in agreement. Combining this with the fact that $J^{(n-1)}=\mathcal{I}$ we conclude that all the nodes in $\mathcal{I}$ are in agreement.

\section{A.2 Proof of Theorem 10}

Notice that despite the existence of a nonzero delay $\tau, \sum_{i=1}^{n} u_{i}=0$. Thus, $\alpha=\operatorname{Ave}(x)$ is an invariant quantity. Given that the solutions of (37) globally asymptotically converge to a limit $x^{*}$, due to the invariance of $\alpha, x_{i}^{*}=\operatorname{Ave}(x(0)), \forall i \in \mathcal{I}$ and the average-consensus will be reached. To establish the stability of (37), we use a frequency domain analysis. We have $X(s)=G_{\tau}(s) x(0)$ where

$$
G_{\tau}(s)=\left(s I_{n}+e^{-\tau s} L\right)^{-1} .
$$

Define $Z_{\tau}(s)=G_{\tau}^{-1}(s)=\left(s I_{n}+e^{-\tau s} L\right)$. We need to find sufficient conditions such that all the zeros of $Z_{\tau}(s)$ are on the open LHP or $s=0$. Let $w_{k}$ be the $k$ th normalized eigenvector of $L$ associated with the eigenvalue $\lambda_{k}$ in an increasing order. For a connected graph $G$, $0=\lambda_{1}<\lambda_{2} \leq \ldots \leq \lambda_{n}=\lambda_{\max }(L)$. Clearly, $s=0$ in the direction $w_{1}$ is a zero of the MIMO transfer function $Z_{\tau}(s)$, because $Z_{\tau}(0) w_{1}=L w_{1}=0$. Furthermore, any eigen vector of $Z_{\tau}(s)$ is an eigen vector of $L$ and vice verse. Let $\left(s, w_{k}\right)$ with $k>1$ be a right MIMO transmission zero of $Z_{\tau}(s)$ at frequency $s$ in the direction $w_{k}$, i.e. $Z_{\tau}(s) w_{k}=0$. Then, $s \neq 0$ satisfies the following equation

$$
s+e^{-\tau s} \lambda_{k}=0,
$$

or

$$
\frac{1}{\lambda_{k}}+\frac{e^{-\tau s}}{s}=0
$$

where $\lambda_{k}$ is the $k$ th eigenvalue of $L$ corresponding to $w_{k}$. This is due to the fact that

$$
Z_{\tau}(s) w_{k}=s w_{k}+e^{-\tau s} L w_{k}=\left(s+e^{-\tau s_{k}} \lambda_{k}\right) w_{k}=0,
$$


but $w_{k} \neq 0$, thus $s+e^{-\tau} \lambda_{k}=0$. Equation (53) provides a Nyquist criterion for convergence of protocol (A2). If the net encirclement of the Nyquist plot of $\Gamma(s)=e^{-\tau s} / s$ around $-1 / \lambda_{k}$ for $k>1$ is zero, then all the zeros of $Z_{\tau}(s)$ (or poles of $\left.G_{\tau}(s)\right)$ ) other than $s=0$ are stable. For the special case where $L$ is symmetric, all the eigenvalues are real and the Nyquist stability criterion reduces to zero net encirclement of the Nyquist plot of $\Gamma(s)$ around $-1 / \lambda_{n}$ (note that $\left.\lambda_{n}=\lambda_{\max }(L)\right)$. This is because the plot of $\Gamma(j \omega)$ in the s-plane remains on the right hand side of $-\tau$. Since

$$
\Gamma(j \omega)=\frac{e^{-j \omega \tau}}{j \omega}=-\frac{\sin (\omega \tau)}{\omega}-j \frac{\cos (\omega \tau)}{\omega}
$$

and clearly $\operatorname{Re}(\Gamma(j \omega))$ is a sinc function satisfying $\operatorname{Re}(\Gamma(j \omega)) \geq-\tau$. A conservative upper bound on $\tau$ can be obtained according to the property $\operatorname{Re}(\Gamma(j \omega)) \geq-\tau$ of the Nyquist plot of $\Gamma(s)$ by setting $-1 / \lambda_{n}>-\tau$ which gives the convergence condition $\tau<1 / \lambda_{n}$. As a by-product, for $\tau=0$, the protocol always converges regardless of the value of $\lambda_{k}, k>1$.

A better upper bound on the time-delay $\tau$ can be calculated as follows. Let us find the smallest value of the time-delay $\tau>0$ such that $Z_{\tau}(s)$ has a zero on the imaginary axis. To do so, set $s=j \omega$ in (52), we have

$$
\begin{aligned}
j \omega+e^{-j \omega \tau} \lambda_{k} & =0 \\
-j \omega+e^{j \omega \tau} \lambda_{k} & =0
\end{aligned}
$$

multiplying both sides of the last two equations gives

$$
\omega^{2}+\lambda_{k}^{2}+j \omega \lambda_{k}\left(e^{j \omega \tau}-e^{-j \omega \tau}\right)=0,
$$

or

$$
\omega^{2}+\lambda_{k}^{2}-2 \omega \lambda_{k} \sin (\omega \tau)=0
$$

Assuming $\omega>0$ (due to $s \neq 0$ ), from (58), we get

$$
\left(\omega-\lambda_{k}\right)^{2}+2 \omega \lambda_{k}(1-\sin (\omega \tau))=0 .
$$

Since both terms in the left hand side of the last equation are positive semi-definite, the equality holds if and only if both terms are zero, i.e.

$$
\begin{aligned}
\omega & =\lambda_{k} \\
\sin (\omega \tau) & =1
\end{aligned}
$$

This implies $\tau \lambda_{k}=2 l \pi+\pi / 2$ for $l=0,1,2, \ldots$, thus the smallest $\tau>0$ satisfies $\tau \lambda_{k}=\pi / 2$. Therefore, we have

$$
\tau^{*}=\min _{\substack{\tau \lambda_{k} \\ k>\pi / 2}}\{\tau\}=\min _{k>1} \frac{\pi}{2 \lambda_{k}}=\frac{\pi}{2 \lambda_{n}}
$$


Due to the continuous dependence of the roots of equation (52) in $\tau$ and the fact that all the zeros of this equation other than $s=0$ for $\tau=0$ are located on the open LHP, for all $\tau \in\left(0, \tau^{*}\right)$, the roots of (52) with $k>1$ are on the open LHP and therefore the poles of $G_{\tau}(s)$ (except for $s=0$ ) are all stable. One can repeat a similar argument for the assumption that $\omega<0$ and get the equation

$$
\left(\omega+\lambda_{k}\right)^{2}-2 \omega \lambda_{k}(1+\sin (\omega \tau))=0
$$

which leads to $\omega=-\lambda_{k}$ and $\tau \lambda_{k}=2 l \pi+\pi / 2$.

For $\tau=\tau^{*}, G_{\tau}(s)$ has three poles on the imaginary axis given by

$$
s=0, s= \pm j \lambda_{n}
$$

All other poles of $G_{\tau}(s)$ are stable and in the steady-state the values of each node takes the following form:

$$
x_{i}^{s s}(t)=a_{i}+b_{i} \sin \left(\lambda_{n} t+\varphi_{i}\right), \quad i \in \mathcal{I}
$$

where $a_{i}, b_{i}, \varphi_{i}$ are constants that depend on the initial conditions.

\section{References}

[1] N. Biggs. Algebraic Graph Theory. Cambridge Tracks in Mathematics. Cambridge University Press, 1974.

[2] I. Daubechies and J. C. Lagarias. Sets of matrices all infinite products of which converge. Linear Algebra and Its Applications, 161:227-263, 1992.

[3] I. Daubechies and J. C. Lagarias. Corrigendum/addendum to: Sets of matrices all infinite products of which converge. Linear Algebra and Its Applications, 327:69-83, 2001.

[4] J. P. Desai, J. P. Ostrowski, and V. Kumar. Modeling and control of formations of nonholonomic mobile robots. IEEE Trans. on Robotics and Automation, 17(6), December 2002.

[5] R. Diestel. Graph Theory, volume 173 of Graduate Texts in Mathematics. SpringerVerlag, 2000.

[6] A. Fax and R. M. Murray. Graph Laplacians and stabilization of vehicle formations. The 15th IFAC World Congress, June 2002.

[7] A. Fax and R. M. Murray. Information Flow and Cooperative Control of Vehicle Formations. The 15th IFAC World Congress, June 2002.

[8] M. Fiedler. Algebraic connectivity of graphs. Czechoslovak Mathematical Journal, 23(98):298-305, 1973. 
[9] M. Fiedler. A property of eigenvectors of nonnegative symmetric matrices and its application to graph theory. Czechoslovak Mathematical Journal, 25(100):619-633, 1975.

[10] F. R. Gantmacher. Matrix Theory, volume II. Chelsea Publishing Company, 1959.

[11] C. Godsil and G. Royle. Algebraic Graph Theory, volume 207 of Graduate Texts in Mathematics. Springer, 2001.

[12] R. A. Horn and C. R. Johnson. Matrix Analysis. Cambridge University Press, 1987.

[13] A. Jadbabaie, J. Lin, and S. A. Morse. Coordination of groups of mobile agents using nearest neighbor rules. IEEE Trans. on Automatic Control (to appear).

[14] R. W. Lawton, J. R. T. Beard and B. J. Young. A Decentralized Approach to Formation Maneuvers. IEEE Trans. on Robotics and Automation (to appear).

[15] N. A. Lynch. Distributed Algorithms. Morgan Kaufmann Publishers, Inc., 1997.

[16] M. Marcus and H. Minc. A Survey of Matrix Theory and Matrix Inequalities. Dover Publications, 1969.

[17] R. Merris. Laplacian matrices of a graph: a survey. Linear Algebra and its Applications, 197:143-176, 1994.

[18] M. Mesbahi. On a dynamic extension of the theory of graphs. Proc. of the American Control Conference, Anchorange, AL, May 2002.

[19] M. Mesbahi and F. Y. Hadegh. Formation flying of multiple spacecraft via graphs, matrix inequalities, and switching. AIAA Journal of Guidance, Control, and Dynamics, 24(2):369-377, March 2000.

[20] R. Olfati Saber and R. M. Murray. Consensus Protocols for Networks of Dynamic Agents. Proc. of the American Control Conference, June 2003.

[21] F. Paganini, J. Doyle, and S Low. Scalable laws for stable network congestion control. Proc. of the Int. Conf. on Decision and Control, Orlando, FL, Dec. 2001.

[22] C. W. Reynolds. Flocks, herds, and schools: a distributed behavioral model. Computer Graphics (ACM SIGGRAPH '87 Conference Proceedings), 21(4):25-34, July 1987.

[23] J. Toner and Y. Tu. Flocks, herds, and schools: A quantitative theory of flocking. Physical Review E, 58(4):4828-4858, October 1998.

[24] T. Vicsek, A. Cziroók, E. Ben-Jacob, and O. Cohen, I. Shochet. Novel type of phase transition in a system of self-deriven particles. Physical Review Letters, 75(6):1226-1229, August, 1995. 
[25] H. Yamaguchi, T. Arai, and G. Beni. A distributed control scheme for multiple robotic vehicles to make group formations. Robotics and Autonomous Systems, 36:125-147, 2001. 